\title{
BMJ Open Improving hearing and vision in dementia: protocol for a field trial of a new intervention
}

\author{
Jemma Regan, ${ }^{1}$ Piers Dawes, ${ }^{2}$ Annie Pye, ${ }^{1}$ Christopher J Armitage,${ }^{3}$ Mark Hann, ${ }^{4}$ \\ Ines Himmelsbach, ${ }^{5}$ David Reeves, ${ }^{4}$ Zoe Simkin, ${ }^{1}$ Fan Yang, ${ }^{4}$ Iracema Leroi ${ }^{1}$
}

To cite: Regan J, Dawes P, Pye $A$, et al. Improving hearing and vision in dementia: protocol for a field trial of a new intervention. BMJ Open 2017;7:e018744. doi:10.1136/ bmjopen-2017-018744

- Prepublication history for this paper is available online To view these files, please visit the journal online (http://dx.doi org/10.1136/bmjopen-2017018744).

Received 19 July 2017 Accepted 24 July 2017

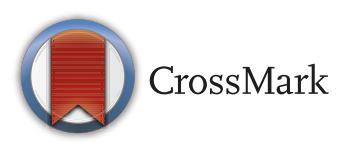

For numbered affiliations see end of article.

\section{Correspondence to} Dr Jemma Regan; jemma.regan@manchester. ac.uk

\section{ABSTRACT}

Introduction Quality of life and other key outcomes may be improved by optimising hearing and vision function in people living with dementia. To date, there is limited research assessing the efficacy of interventions aimed at improving hearing and vision in people with dementia. Here, we outline a protocol to field test a newly developed home-based intervention, designed to optimise sensory functioning in people with dementia in three European sites. The results of this study will inform the design and conduct of a full-scale randomised controlled trial (RCT) in five European sites.

Methods and analysis In this multisite, single arm, open label, feasibility study, participants with dementia $(n=24)$ will be assessed for hearing and vision impairments and be prescribed a hearing aid and/or glasses. Each participant will have a study partner ('dyads'). A subset of dyads will receive 'sensory support' from a 'sensory support therapist', comprising home visits over 12 weeks. The therapist will offer the following intervention: adherence support for corrective devices; adaptations to the home environment to facilitate sensory function; communication training; and referral to communitybased support services. The primary outcomes will be process measures assessing the feasibility, tolerability and acceptability of: (1) the intervention components; (2) the method of implementation of the intervention and (3) the study procedures, including outcome assessment measures. Quantitative data will be collected at baseline and follow-up. Qualitative data using semistructured interviews will be collected postintervention and weekly, using participant diaries. Finally, we will explore a model of cost-effectiveness to apply in the subsequent fullscale trial. This feasibility study is a necessary step in the development of a complex, individualised, psychosocial intervention. The data gathered will allow logistical and theoretical processes to be refined in preparation for a full-scale RCT.

Ethics and dissemination Ethical approval was obtained in all three participating countries. Results of the field trial will be submitted for publication in a peer-reviewed journal.

\section{INTRODUCTION}

In Europe, there are approximately 10.5 million people living with dementia. ${ }^{1}$ Furthermore, over two-thirds of older

\section{Strengths and limitations of this study}

- This study is limited by being adaptable to the pragmatic realities of a multicountry project while maintaining scientific integrity. For example, in terms of understanding different health organisations and ethics processes, monitoring accurate translation of questionnaire scales, permissions and copyright.

- This study is led by experienced research leaders across the fields of psychiatry, psychology, optometry and audiology.

- This study has an integrative, iterative approach to the research programme; testing the feasibility and operational aspects of the sensory intervention aims to enable a more robust, subsequent randomised controlled trial.

Europeans experience age-acquired sight or hearing loss. ${ }^{2}$ Presbycusis is one of the most common age-related conditions ${ }^{3}$ and the rate of self-reported hearing difficulties increases in the older adult population with the onset of dementia. ${ }^{4}$ For the general population aged 75 years and above, one in four people experience sight loss and for those people aged 85 years and above this rises to one in three people. ${ }^{5}$ Sight loss in dementia is more prevalent than in healthy older adult populations. ${ }^{6}$ Despite the prevalence of sensory impairment in dementia, hearing and vision loss is often not diagnosed and not treated. ${ }^{7}$

The comorbidity of sensory impairment and dementia has been associated with an increased mortality rate in the elderly, ${ }^{8}$ and hearing and vision deficits are independent risk factors for increased cognitive impairment, lower quality of life, anxiety and depression in people with dementia. ${ }^{9-14}$ The higher prevalence of depression and disorientation may lead to isolation from family, loss of social activities and hobbies and marginalisation within the community, as the person is less able to use visual and auditory cues to orientate themselves and communicate. $^{15-17}$ Caregiver burnout is compounded 
by communication barriers and a greater dependency of self-care for activities of daily living. ${ }^{18}$

There is a small existing literature regarding the application of hearing and vision rehabilitation in older people with sensory impairments, ${ }^{19-21}$ but this does not comprehensively extend to people who have concurrent cognitive impairment, particularly dementia. ${ }^{22}$ Despite this, there is some preliminary evidence that sensory remediation in dementia can be effective in the following ways: alleviating personal and social difficulties ${ }^{23}$; decreasing the impact of behavioural and psychological symptoms of dementia ${ }^{24}$; improving cognition with hearing aid use ${ }^{2526}$ and reducing social isolation. ${ }^{27}$ Nonetheless, to be effective, treatment should be introduced at an early stage in the condition ${ }^{28}$ and should be tailored to suit the specific care needs of the individual. ${ }^{29}$ For example, optometric assessments may need to be adapted for older people with dementia to account for the impact of impaired decision-making and the fluctuation in cognitive ability and mental capacity. ${ }^{30}$

Psychosocial therapies offer a potential solution for improving quality of life for people with dementia and concurrent sensory impairment. Here, we outline a protocol to field test the feasibility and tolerability of a newly developed home-based, individualised intervention, with psychosocial aspects, designed to support hearing and vision functioning in people with dementia. The results of this field trial will inform the design and conduct of a full-scale randomised controlled trial (RCT) across several European sites evaluating the efficacy of this 'sensory support intervention' (SSI) on quality of life and other outcomes in people with dementia. This study is part of a wider programme: the 'SENSE-Cog project'.

\section{Theoretical basis for a new intervention}

The new intervention being field trialled here, the 'SENSE-Cog SSI', is based on the premise that improving sensory deficits through corrective devices alone may not be sufficient to improve outcomes for people with dementia. Instead, the SSI aims to offer a period of sustained support delivered by a sensory support therapist (SST) to participants, to assist them to achieve positive, lasting changes in behaviour, as a result of optimised sensory rectification. This support is as follows: continuous training in use of glasses and hearing aids; promoting a 'sensory friendly' home environment; delivering communication techniques and assisting with access to community support services. It is anticipated these SSI components will lead to a change in behaviour by improving uptake of glasses and hearing aids through daily wear and second, improving the persons quality of life (measured by DemQol and DemQol Proxy), ${ }^{31}$ for example, by participating in activities which may have been previously hindered or avoided, as a result of sensory impairment. Encouraging a change in behaviour can be challenging and evidence has shown that many attempted behaviour changes may not be sustained, unless key underlying elements are addressed. ${ }^{32}$ In this study, the underlying elements are existing attitudes and knowledge about participants' own sensory impairment and towards sensory equipment, contributing to subsequent behaviours such as avoiding social situations or impaired communication. Consequently, we have developed our SSI using an evidenced-based model of behavioural change, the COM-B model ${ }^{33}$ as the theoretical framework. Each component of the SSI aligns with each domain of the COM-B model, as outlined in table 1 : 'capability' (C); 'opportunity' $(\mathrm{O})$ and 'motivation' and aims to promote a sustained behavioural change (B) in the appropriate usage of sensory equipment and living well with dementia and sensory impairment (see table 1).

\section{RESEARCH QUESTION}

\section{Overall research question}

Is a newly developed, individualised, sensory support intervention for people with dementia and concurrent sensory impairment suitable for full-scale efficacy testing in a subsequent RCT in different European sites?

\section{Specific research questions for the feasibility study}

- What is the optimal manner to implement an SSI in the participant group?

- What is the acceptability and tolerability of the SSI by participants?

- What specific local contextual issues should be considered in delivering a full trial across multiple different EU sites?

- Is it possible to use the health economics data in a cost effectiveness model?

\section{METHODS AND ANALYSIS \\ Research design}

This is a single-arm, open-label field study with no control group, to assess the feasibility, acceptability and tolerability of a new sensory support intervention for people with dementia $(\mathrm{PwD})$ and concurrent hearing and/or vision impairment.

\section{Study procedures}

We will recruit 24 participant dyads, each comprising a PwD and their study partner, across three clinical sites (eight dyads per site) in Manchester, UK; Bordeaux, France and Nicosia, Cyprus. Following a full hearing and vision assessment, each participant will receive a hearing aid and/or glasses, if required, alongside either basic support of sensory equipment in an 'abbreviated' intervention (components 1 and 2) over three to four visits across 3-4 weeks or 'extended' support ( $n=4$ participants in Manchester). The extended intervention will be delivered to participants by a research therapist, the SST. The research therapist has experience in delivering supportive interventions to people with long-term conditions and will receive additional training in hearing and vision rehabilitation. Starkey Hearing Technologies will provide an operations manual for the provision of 
Table 1 The capability, opportunity, motivation-behavioural model and the corresponding components of the sensory support intervention leading to anticipated behavioural change

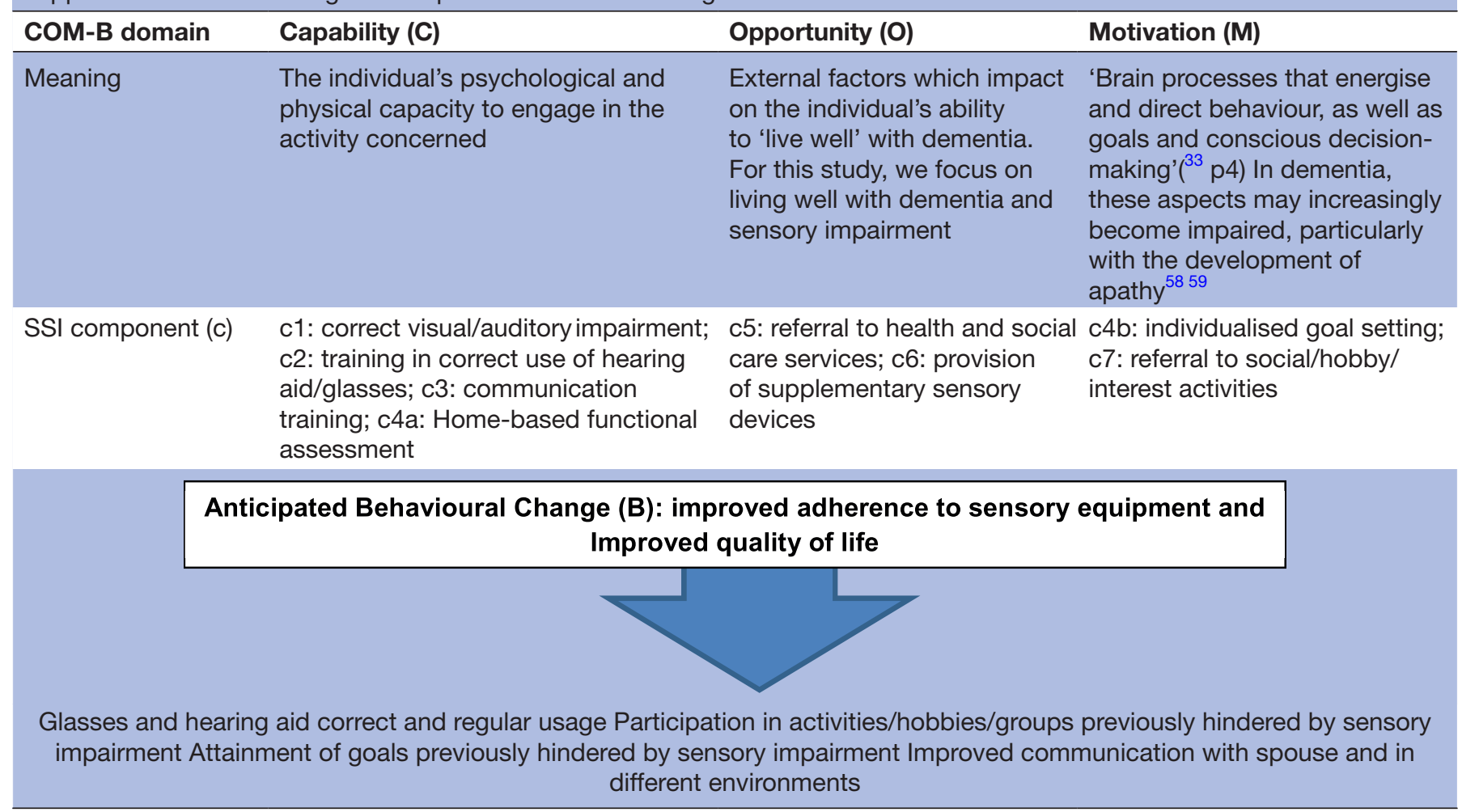

hearing devices and advice on care specific to the device. They will also provide training to the SST on the correct maintenance and cleaning of the hearing aid. The abbreviated version of the intervention will allow testing of the 'logistics' circuit' for the supply of the corrective devices (hearing aids and glasses), the scheduling of the hearing and vision assessments, the timing of the baseline and end-point outcome evaluations, the operations of the recruitment and retention strategy and the tolerability of the study protocol and procedures. Additionally, at the Manchester site, the extended intervention (components 3 to 7 ) will enable us to evaluate each component of the intervention and the fidelity of delivery of the intervention by the SST. After each visit, the PwD, study partners and the SST will complete a diary to capture experiences, acceptance, tolerability and adherence to procedures. A subset of participants $(n=15)$ will undertake a semi-structured interview postintervention to ascertain perceptions and experiences of the intervention. The schedule of study procedures is outlined in table 2 .

\section{Study sample $\mathrm{e}^{34}$}

Participants with dementia: inclusion criteria

To be included in the study, each person with dementia must meet the following inclusion criteria:

- Age 60 years or older;

- Has a formal, clinical diagnosis of dementia of the following subtypes: Alzheimer's disease (AD), as per National Institute of Neurological and Communicative Disorders and Stroke and the Alzheimer's
Disease and Related Disorders Association (NINCDSADRDA) criteria $^{35}$; vascular dementia (VaD) or mixed $\mathrm{AD} / \mathrm{VaD}$;

- Montreal Cognitive Assessment (MoCA) ${ }^{35}$ score of 12 or above;

- Adult acquired hearing and/or vision impairment;

- Hearing threshold $>35 \mathrm{~dB}$ HL over $1-3 \mathrm{kHz}$ and/ or vision score of present binocular corrected visual acuity of $\leq 6 / 9,5$ Snellen metric or $+0.2 \operatorname{LogMAR}(75$ Early Treatment Diabetic Retinopathy Study (ETDRS) Score) and/or visual field of $10-20^{\circ}$;

- Speaks and understands the language of the intervention delivery;

- Is willing to accept sensory support;

- Is living in an ordinary community dwelling (including sheltered and very sheltered accommodation);

- Has a study partner willing to participate in the study (a family member or close friend who is either coresident or in regular contact (at least three times per week);

- Has mental capacity sufficient to give informed consent to participate.

Participants with dementia: exclusion criteria

If the person with dementia has one or more of the following, they will be excluded from participation in the study:

- Congenital hearing or vision impairment; 


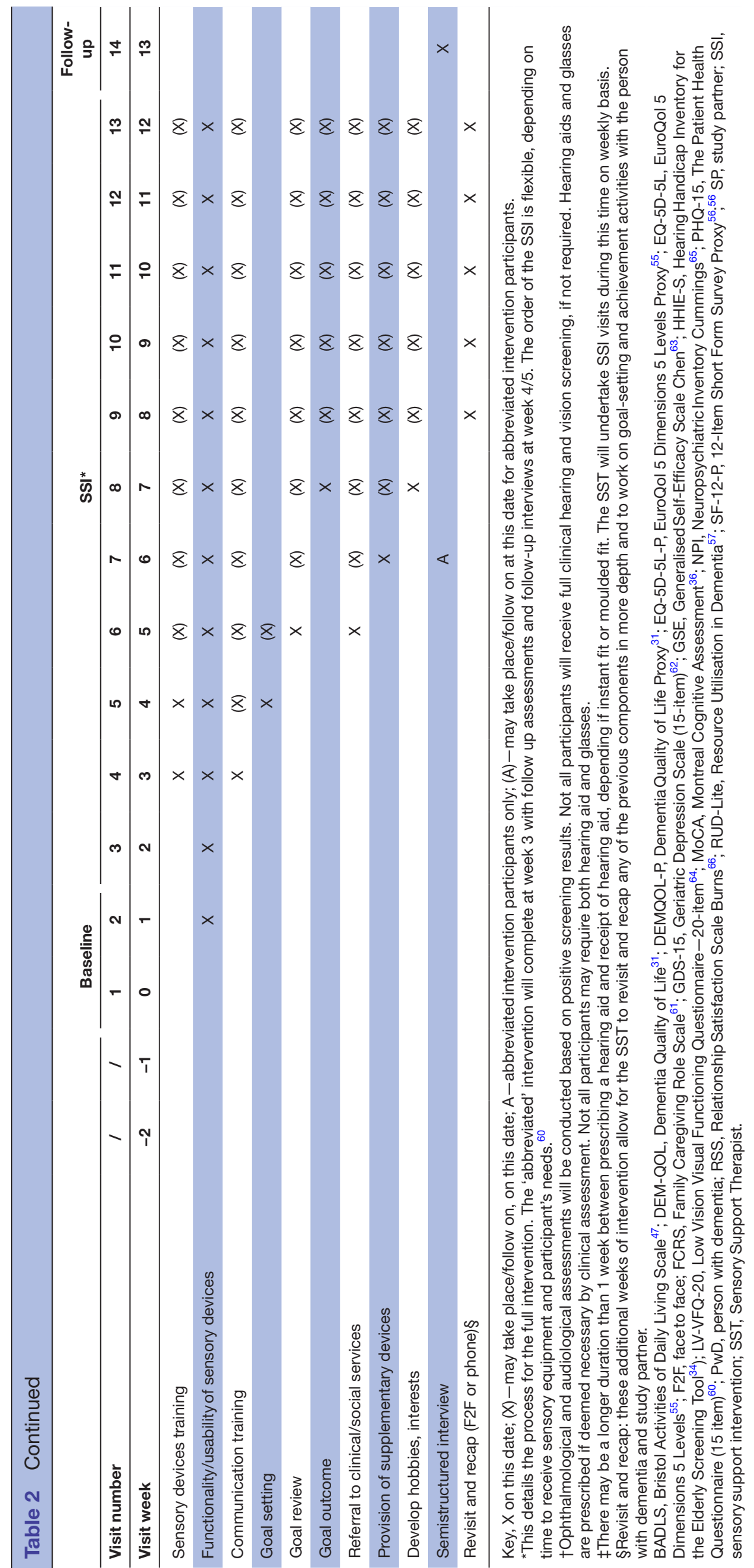


- Unstable, acute and current psychiatric or physical condition severe enough to prevent them from undertaking the study procedures;

- Has a less common form of dementia (eg, Parkinson's disease dementia, dementia with Lewy bodies, frontotemporal dementia);

- Is currently participating in any other medication or non-medication related trial;

- Has urgent treatment scheduled for hearing or vision (eg, cataract operation, treatment for macular degeneration needed).

\section{Study partner: inclusion criteria}

To be included in the study, the study partner must meet the following inclusion criteria:

- Age 18 years or older;

- Speaks and understands language of intervention delivery;

- Able to read and write;

- Not employed as a professional carer for the PwD, (except Nicosia, which may include professional, live-in carers);

- Is a family member or a close friend who is either coresident or in regular contact (minimum of three times per week).

\section{Study partner: exclusion criteria}

If the person with study partner has the following, they will be excluded from participation in the study:

- Has an unstable, acute and current psychiatric or physical condition severe enough to prevent them from participating.

\section{Screening methods}

Cognition will be screened by administering the MoCA. ${ }^{36}$ Hearing will be screened with a simple handheld screening device, 'HearCheck' ${ }^{37}$ Vision will be screened using the 'PEEK Acuity App'38 alongside the confrontation visual field test: 'can you see my hands?'. ${ }^{39}$

\section{Recruitment}

Recruitment will occur over a 3-month period, beginning Spring 2017. In all three sites, participants will be recruited from local memory clinics and dementia research registries. Ethically approved participant information sheets (PIS) will be provided to inform potentially eligible participants about the study. At least 24 hours will be given for the potential participants to read and understand the PIS, prior to being offered a screening appointment. Informed, written consent will be obtained prior to participation and following an assessment of capacity to consent to the study.

\section{The intervention}

The SSI to be field tested was developed in accordance with the UK's Medical Research Council's (MRC) guidelines on the development and testing of complex interventions. ${ }^{40}$ This involved a multistage programme which generated data that were synthesised to develop the current version of the intervention. Details of the this development programme are reported elsewhere ${ }^{41}$ and involved the following: (1) scoping the gaps in understanding, awareness and service provision for the hearing and/or vision impairment care needs of people with dementia, using a systematic literature review $^{42}$ and an expert reference group; (2) investigating the support care needs through a literature search, stakeholder survey, focus groups, semistructured interviews and an expert reference group, leading to a prototype sensory support package; (3) refining the prototype through additional input from stakeholders using focus groups and semistructured interviews. Input from lay members (the 'patient and public voice') is a cornerstone of the work and informed the development process at each step. ${ }^{43}$

\section{Components of the intervention}

The complete intervention comprises seven components. For this field trial, an abbreviated version of the intervention (the abbreviated intervention comprising only components 1 to 2) will be administered in the study sites in Nicosia and Bordeaux. In the Manchester site, the full intervention (the extended) will be administered.

\section{Abbreviated intervention}

Following screening, participants at all three sites will be administered the following intervention components:

\section{Component 1 : identify and correct any vision or hearing impairment}

Vision and hearing assessments will be undertaken by an audiologist, optometrist or ophthalmologist, according to standardised procedures (see table 3 ).

Should medical management of cataracts or macular degeneration be necessary following this visual assessment, participants will be deemed ineligible for the study and referred for medical or surgical management as per standard care. Essilor International ${ }^{44}$ will provide the lenses for the study. Starkey Hearing Technologies ${ }^{45}$ (based in Stockport, UK) will provide the hearing aids. These will be the 'behind the ear' fitting Muse Mini BTE i2400.

\section{Component 2: continuous training in the correct use of sensory devices}

The SST will work with participants to ensure adherence and correct usage of the prescribed hearing aids and glasses. For example, advice will be given on cleaning and storing devices correctly and changing batteries as required (based on a checklist provided by Essilor Limited and Starkey Hearing Technologies).

\section{Extended intervention}

Following components 1 and 2, each participant in the Manchester site will be contacted by the research therapist, the SST, to undertake the remaining components of the intervention over a 12-week period, with a maximum of 12 weekly, one 2-hour sessions in the participants' home (see table 2). Each intervention step will be tailored to meet the participant's individual needs, which will be 
Table 3 Audiological and ophthalmological examination procedures

\begin{tabular}{ll}
\hline Audiology examination & Ophthalmological examination \\
\hline Medical and social history & Current optical correction \\
Otoscope & Habitual vision/habitual Visual Acuity (both LogMAR and Snellen equivalent) \\
\hline Ambient noise evaluation & Medical and vision history \\
Pure tone audiometry & Intraocular pressures \\
Uncomfortable loudness levels examination & Ocular motor balance assessment \\
Glasgow Hearing Aid Benefit Profile ${ }^{67}$ & Ocular health assessment using direct ophthalmoscopy \\
& Fundus \\
& Subjective prescription findings \\
& Objective prescription findings using retinoscopy \\
& Visual field testing \\
\hline
\end{tabular}

considered from multiple perspectives (environment, existing knowledge and skills and access to services). The remaining five components (components 3 to 7 ) may be delivered in a flexible order.

\section{Component 3: communication training}

Communication difficulties are a barrier to living well with dementia and sensory impairment. ${ }^{46}$ Based on findings from our focus group with occupational therapists from across Europe (step 2 of our development programme), communication difficulties between a person with dementia and sensory impairment and their spouse was a prominent theme. The SST will work with dyads on improving communication, using adapted, evidence-based materials.

Component 4: home-based functional assessment and goal setting During baseline assessments, the PwD will complete the Bristol Activities of Daily Living Scale, ${ }^{47}$ a homebased functional assessment of activities of daily living. Combined with the results of the hearing and/or vision assessment, this will form the basis for setting personalised goals by the participant and their study partner, supported by the SST. Goal setting is a highly successful strategy for use in this population. ${ }^{48}$ Goals will be revisited by the SST on a weekly basis. The Bangor Goal Setting Inventory, which reviews goal attainment at 25\%, $50 \%$, $75 \%$ and $100 \%$ increments, ${ }^{49}$ will be the tool used by the SST to assist the participant dyad with goal setting and monitoring achievement of goals.

\section{Component 5: referral to health and social services}

Based on the functional assessment and goals set in component 6 , the SST may refer participants to health or social care services in the community. This may include psychological services, geriatric psychiatry services, falls' clinics or other services, as indicated by the assessments and goal-setting exercises. This will be particularly important for participants who may be socially isolated and not in contact with supporting agencies.
Component 6: provision of supplementary sensory devices to enhance the home environment

Participants with visual impairment will be offered a lamp (The Daylight CompanyTwist Portable Lamp in White D33700) to assist with low vision in the home setting. Glasses' straps and hearing aid clips will be provided, if required. Participants will be signposted to beneficial devices to support vision and hearing function in the home, which may correspond with their goal setting.

Component 7: fostering social inclusion through hobbies/interests/ social groups

People with dementia and sensory impairment may become isolated from family and the wider community. ${ }^{51}$ Thus, it is important to link dyads to community groups and resources, based on their hobbies and interests. Information about support for hobbies and interests will be sourced from local charities and healthcare providers.

\section{Component 8: recap and revisit}

The remaining weeks allow the SST to revisit and recap previous components, based on the participant's individual needs and goal-attainment.

\section{Outcome measures}

\section{Primary outcome measures}

A set of process measures will be undertaken to assess the primary outcomes of the study: are the intervention components, method of implementation and study processes are feasible, acceptable and tolerated? This includes an assessment of the following: recruitment; retention; screening procedure; inclusion/exclusion criteria; assessment of impairment; the role of support offered by the SST; delivery and fitting of devices and the functional assessment procedure. We will also include measures of behavioural change, as per the COM-B approach (capability, opportunity and motivation) regarding the use of hearing aids and glasses, captured by the diaries and semistructured interviews (see table 4 ). 
Table 4 SENSE-Cog sensory support intervention field study primary outcomes measures and methods to capture outcomes

\begin{tabular}{|c|c|}
\hline Field study outcome & Method to capture outcome \\
\hline $\begin{array}{l}\text { PwD effort } \\
\text { PwD fatigue } \\
\text { PwD motivation } \\
\text { PwD engagement } \\
\text { PwD understanding }\end{array}$ & $\begin{array}{l}\text { In-house rating scale in PwD and SP diaries; semistructured interview with both } \\
\text { dyad members }\end{array}$ \\
\hline SSI feasibility & Completion rates/missing data at baseline and follow-up \\
\hline Is SSI delivered as intended? & SST diary checklist after each visit \\
\hline Is SSI received as intended? & $\begin{array}{l}\text { Records of contact between SST and recipient. This will include information } \\
\text { on: number and duration of contact, sessions; method; referrals and protocol } \\
\text { deviations. } \\
\text { PwD and SP will have their knowledge of the SSI components checked by the } \\
\text { SST }\end{array}$ \\
\hline Recruitment & Number of patients approached versus number recruited \\
\hline Retention & Number of participants withdrawing and reasons \\
\hline Screening & Number of patients screened 'suitable' versus 'unsuitable' \\
\hline Inclusion/exclusion criteria & Feedback from CRN/other recruiters about ease of screening criteria \\
\hline $\begin{array}{l}\text { Assessment of impairment and corrective } \\
\text { device delivery and fitting }\end{array}$ & SST, PwD, SP diaries; feedback from research team and industry partners \\
\hline Functional assessments & SST, PwD, SP diaries; analysis of frequency of missing data \\
\hline $\begin{array}{l}\text { 'Logistics circuit' of hearing/ } \\
\text { vision assessments and devices }\end{array}$ & SST, PwD, SP diaries; feedback from research team and industry partners \\
\hline Helpfulness of SSI & \\
\hline Motivation to continue with SSI activities & \\
\hline
\end{tabular}

CRN, Clinical Research Network; PwD, people with dementia; SP, study partner; SSI, sensory support intervention; SST, sensory support therapist.

\section{Secondary outcome measures}

The secondary outcome is to gain an initial impression of treatment efficacy and ascertain the utility of different ratings scales. We will examine preintervention (baseline) and postintervention (follow-up) measures using a battery of rating scales of the following dementia-related outcomes, completed by the $\mathrm{PwD}$ and study partner: quality of life; behaviour/mood; general mental wellbeing; functional ability; mental health; social connectedness; burden and stress due to the caregiver role and caregiver/patient relationship. It is important to include economic evaluation during field testing to ascertain utility for the subsequent RCT $^{52}$ and we will assess the use of the quality of life and healthcare resource instruments. For full details of scales corresponding to field study outcome, see table 5 .

\section{Data analysis}

\section{Qualitative analysis}

The qualitative analyses of the semistructured interviews and diaries will highlight aspects of perceived efficacy. Data from the diaries and verbatim data from the interviews will be analysed using summative content analysis. ${ }^{53}$ The findings will be analysed into codes and descriptive evidence to support the coding framework. A subsection of interview sessions $(n=15)$ will be audiotaped, transcribed verbatim and analysed using MaxQda qualitative software $^{54}$ by colleagues at University of Freiburg, Germany. The diary material will be analysed by the University of Manchester. Based on summative content analysis, we will formulate criterion from the theoretical background and the research question. This criterion will be as follows: the perception of the intervention material; adherence 
Table 5 SENSE-Cog SSI field study secondary outcomes measures and methods to capture outcomes

\section{Tool to capture outcome}

\begin{tabular}{|c|c|}
\hline \multicolumn{2}{|l|}{ PwD outcome } \\
\hline Quality of life & Dementia Quality of Life ${ }^{31}$; EuroQol 5 Dimensions 5 Levels $^{55}$; 12-Item Short Form Survey ${ }^{56}$ \\
\hline Cognition & Neuropsychiatric Inventory ${ }^{65}$ \\
\hline Function & Bristol Activities of Daily Living Scale ${ }^{47}$ \\
\hline Cognition & Montreal Cognitive Assessment ${ }^{36}$ \\
\hline Quality of Life & $\begin{array}{l}\text { Dementia Quality of Life Proxy }{ }^{31} \text {; EuroQol } 5 \text { Dimensions } 5 \text { Levels Proxy }{ }^{55} \text {; } 12 \text {-Item Short Form } \\
\text { Survey Proxy }{ }^{56}\end{array}$ \\
\hline Mental health & Geriatric Depression Scale $15^{62}$ \\
\hline Burden and stress & Family Caregiving Role Scale ${ }^{61}$ \\
\hline Healthcare resource use & Resource Utilisation in Dementia Lite ${ }^{57}$ \\
\hline
\end{tabular}

PwD, person with dementia; SP, study partner; SSI, sensory support intervention

to the glasses; hearing aids and sensory support, documented in terms of behavioural change. We will deduce the categories in an iterative manner, based on data from the semistructured interviews and diaries. Within a feedback loop, the identified categories will be revised and refined in to the main themes and checked against the data for reliability.

\section{Quantitative analysis}

For this feasibility study, we did not perform a formal power calculation. Data will be entered into the Statistical Package for the Social Sciences V.20.0.0.2 (SPSS) and recoded so all items have a consistent valance. Any missing data will be recorded, along with any protocol violations. To explore participants' impression of change across various outcome parameters (eg, quality of life, cognition, behaviour, social connectedness, mental wellbeing, study partner indices), we will examine the difference (ie, change) between baseline (preintervention) and follow-up (postintervention) subjective rating scales of 'perceived efficacy' to explore the feasibility, tolerability and face validity of the measures for the subsequent RCT. The distributions of the secondary outcome measures, both preintervention and postintervention, will be summarised using appropriate measures of central tendency (mean or median) and variability (SD or IQR). Due to the small sample, we will use a large $p$ value, 0.2 , as an indication of effect only. The score distributions, both preintervention and postintervention, will be examined for degree of variability and ceiling and floor effects.

\section{Economic analysis}

We will summarise the statistics of EuroQoL 5 Dimensions (EQ-5D), ${ }^{55}$ 12-Item Short Form Survey (SF-12) ${ }^{56}$ and Resource Utilisation in Dementia (RUD)-Lite. ${ }^{57}{\text { EQ- } 5 D^{55}}^{55}$ and EQ-5D Proxy ${ }^{55}$ version will allow health utility data to estimate quality-adjusted life years (QALYs). Costs will be calculated based on the number of healthcare resources used, collected using RUD-Lite and the reference costs estimated from published reports, for example, Personal Social Services Research Unit unit costs, administrative datasets and/or experts' opinion. We will conduct exploratory modelling work using preintervention and postintervention health economics data to ensure it is possible to use these data in a cost-effectiveness model. SF-12-based and Dementia Quality of Life-derived health utility data will be implemented as alternative ways to calculate QALYs.

\section{Ethics and dissemination}

The study has received favourable ethical approval for Manchester on 30th September 2016 from the NW Preston REC IRAS ID 212501, REC reference 16/ NW/0685 and the Health Research Authority on 29th November 2016. For Nicosia, the study was reviewed by the Cyprus National Bioethics Committee on the 27th September 2016 and bioethical approval was granted. For Bordeaux the ethical opinion from the Comité de Protection des Personnes Sud-ouest et Outremer III was given on 25th May 2016.

Manuscripts with the results of this field trial will be submitted for publication in peer-reviewed journals. We will post results on the SENSE-Cog website (www.sense (www.sense-cog.eu/) and on the SENSE-Cog Twitter account (@sense_cog). Feedback will be sent to the RCT Trial Development Team and disseminated at public engagement events.

\section{DISCUSSION}

This field trial is the next logical step in a multipart process, developing a new, complex intervention. The field trial was 
designed to refine and test the feasibility, tolerability and acceptability of a draft intervention to support hearing and vision functioning in PwD living at home across Europe. Data from this field trial will highlight weaknesses and strengths in the components and implementation of the intervention, as well as the study procedures and conduct in preparation for a five-site, full-scale RCT beginning Autumn 2017. This field trial will also evaluate the efficacy of a new job role designed specifically for the SENSE-Cog research, the SST; allow refinement of the SST training, SST job description and step-by-step user manuals across sites prior to the RCT. While the RCT will test the efficacy of the SSI and impact on quality of life for people with dementia and study partners, trialling of these operational aspects 'in the field' prior to the RCT is imperative to ensure these can be optimally implemented in a larger scale, complex, multisite, cross-disciplinary study.

The outcome of the subsequent RCT aims to expound the benefits of a domiciliary approach for hearing and vision assessments and sustained support for people with dementia. This sensory support package is derived from existing local resources, driven by person-centred goals and could potentially lessen the economic impact on EU clinical services, by offering a home-based, individualised alternative to a pharmacological model of care for dementia and sensory loss.

\section{Study timeline}

The overall programme started on 1st January 2016. The Expert Reference was held in Athens, Greece in April 2016. Applications for ethical approval were submitted and favourable approval received in August and September 2016. This study will begin recruiting in Spring 2017 and will be completed by September 2017. The RCT to follow this development programme is scheduled to begin in November 2017.

\section{ACKNOWLEDGEMENTS}

The authors thank Lucas Wolski at the University of Freiburg for assisting with analysis and design. We thank the industry collaborators at The Outside Clinic, Starkey Hearing Technologies, Esillor International, Siemens Hearing Aids, Sivantos Group, PEEK Vision and HearCheck. We thank Joshua Woods for input in the development of the sensory support intervention and Brenda Gannon for health economics input. We thank the Greater Manchester NIHR Clinical Research Network for supporting the study. We thank patients and their spouses from the Research User Group for input on the design and development of the intervention.

\section{Author affiliations}

${ }^{1}$ Division of Neuroscience and Experimental Psychology, University of Manchester and the Manchester Academic Health Sciences Centre, Manchester, UK ${ }^{2}$ Manchester Centre for Audiology and Deafness (ManCAD), University of Manchester and the Manchester Academic Health Sciences Centre, Manchester, U.K ${ }^{3}$ Manchester Centre for Health Psychology, School of Psychological Sciences, Manchester Academic Health Science Centre, University of Manchester, Manchester, UK
${ }^{4}$ Division of Population Health, Health Services Research and Primary Care, School of Health Sciences, Manchester Centre for Health Economics, University of Manchester, Manchester, UK

${ }^{5}$ Institute of Applied Research, Development and Continuing Education, Catholic University of Applied Sciences, Freiburg, Germany

\section{Twitter @sense_cog}

Acknowledgements The authors thank Lucas Wolski at the University of Freiburg for assisting with analysis and design. We thank industry collaborators at The Outside Clinic, Starkey Hearing Technologies, Esillor International, Siemens Hearing Aids, Sivantos Group,Peek Vision and HearCheck. We thank Joshua Woods for input in the development of the Sensory Support Intervention and Brenda Gannon for health economics input.We thank the Greater Manchester NIHR Clinical Research Network for supporting the study. We thank patients and their spouses from the Research User Group for input on the design and development of the intervention.

Contributors IL and PD are the programme leads and conceptualised and designed the field trial. JR is study coordinator and had primary responsibility for writing the paper. ZS and AP are research assistants and FY provided health economic input. MR and DR provided statistical input for the study. CA is a health psychologist and provided input on the theoretical framework. $\mathrm{IH}$ is leading the qualitative methods and analysis. All authors were involved in critical revision of the article.

Funding This work was supported by the European Union's Horizon 2020 research and innovation programme under grant agreement № 668648.

Competing interests None declared.

Patient consent Obtained.

Ethics approval North West—Preston—Health Research Authority.

Provenance and peer review Not commissioned; peer reviewed for ethical and funding approval prior to submission.

Open Access This is an Open Access article distributed in accordance with the Creative Commons Attribution Non Commercial (CC BY-NC 4.0) license, which permits others to distribute, remix, adapt, build upon this work non-commercially, and license their derivative works on different terms, provided the original work is properly cited and the use is non-commercial. See: http://creativecommons.org/ licenses/by-nc/4.0/

(c) Article author(s) (or their employer(s) unless otherwise stated in the text of the article) 2017. All rights reserved. No commercial use is permitted unless otherwise expressly granted.

\section{REFERENCES}

1. Prince M, Wimo A, Guerchet M, et al. World Alzheimer Report. London: Alzheimer's Disease International, 2015.

2. Wittchen HU, Jacobi F, Rehm J, et al. The size and burden of mental disorders and other disorders of the brain in Europe 2010. Eur Neuropsychopharmacol 2011;21:655-79.

3. Roth TN, Hanebuth D, Probst R. Prevalence of age-related hearing loss in Europe: a review. Eur Arch Otorhinolaryngol 2011;268:1101-7.

4. Zekveld AA, George EL, Houtgast T, et al. Cognitive abilities relate to self-reported hearing disability. J Speech Lang Hear Res 2013;56:1364-72.

5. Owen CG, Fletcher AE, Donoghue M, et al. How big is the burden of visual loss caused by age related macular degeneration in the United Kingdom? Br J Ophthalmol 2003;87:312-7.

6. Bowen M, Edgar DF, Hancock B, et al. The Prevalence of Visual Impairment in People with Dementia (the PrOVIDe study): a cross sectional study of 60-89 year old people with dementia and qualitative exploration of individual, carer and professional perspectives. Health Services and Delivery Research. Southampton: National Institute of Health Research 2016. No. 4.21.

7. McGilton KS, Höbler F, Campos J, et al. Hearing and vision screening tools for long-term care residents with dementia: protocol for a scoping review. BMJ Open 2016.

8. Mitoku K, Masaki N, Ogata Y, et al. Vision and hearing impairments, cognitive impairment and mortality among long-term care recipients: a population-based cohort study. BMC Geriatr 2016;16:112.

9. Evans JR, Fletcher AE, Wormald RPL. Age-Related Macular Degeneration Causing Partial sight and blindness in People 75 Years or Older in Britain. Ophthalmology 2004b;111:513-7. 
10. Lin FR, Metter EJ, O'Brien RJ, et al. Hearing loss and incident dementia. Arch Neurol 2011;68:214-20.

11. Parham K, Lin FR, Coelho DH, et al. Comprehensive management of presbycusis: central and peripheral. Otolaryngol Head Neck Surg 2013;148:537-9.

12. Peters CA, Potter JF, Scholer SG. Hearing impairment as a predictor of cognitive decline in dementia. J Am Geriatr Soc 1988;36:981-6.

13. Strawbridge WJ, Wallhagen MI, Shema SJ, et al. Negative consequences of hearing impairment in old age: a longitudinal analysis. Gerontologist 2000;40:320-6.

14. Valentijn SA, van Boxtel MP, van Hooren SA, et al. Change in sensory functioning predicts change in cognitive functioning: results from a 6-year follow-up in the maastricht aging study. J Am Geriatr Soc 2005:53:374-80.

15. McKeefry D, Bartlett R. Improving vision and eye health care for people with dementia: Thomas Pocklington Trust, 2010. Number 8. http://www.pocklingtontrust.org.uk/Resources/Thomas\% 20Pocklington/Documents/ PDF/Research\%20Publications/RDP8. pdf

16. Elliott $A F$, McGwin G, Owsley C. Vision-enhancing interventions in nursing home residents and their short-term effect on physical and cognitive function. J Am Geriatr Soc 2009;57:202-8.

17. Lupsakko T, Mäntyjärvi $\mathrm{M}$, Kautiainen $\mathrm{H}$, et al. Combined hearing and visual impairment and depression in a population aged 75 years and older. Int J Geriatr Psychiatry 2002;17:808-13.

18. Lawrence V, Murray J, Banerjee S, et al. The experiences and needs of people with dementia and serious visual impairment: a qualitative study, Thomas Pocklington Trust occasional paper 16. London: Thomas Pocklington Trust 2008.

19. Acton JH, Molik B, Court H, et al. Effect of a home Visit-Based Low Vision Rehabilitation Intervention on Visual Function Outcomes: An Exploratory Randomized Controlled Trial. Invest Ophthalmol Vis Sci 2016:57:6664-7.

20. Wong LL, Yu JK, Chan SS, et al. Screening of cognitive function and hearing impairment in older adults: a preliminary study. Biomed Res Int 2014;2014.

21. Kreeger JL, Raulin ML, Grace J, et al. Effect of hearing enhancement on mental status ratings in geriatric psychiatric patients. Am J Psychiatry 1995;152:629-31.

22. Dawes $P$, Wolski $L \mathrm{H}$, et al. Interventions for hearing and vision impairment to improve outcomes for people with dementia: A systematic review. Int Psychogeriatr;2017.

23. Rogers MA, Langa KM, Rogers SL, et al. Untreated poor vision: a contributing factor to late-life dementiaNatural history of branch retinal vein occlusion: An evidence-based systematic review. Am J EpidemiolOphthalmology 2010;117:1094-101.

24. Martini A, Castiglione A, Bovo R, et al. Aging, cognitive load, dementia and hearing loss. Audiol Neurootol 2014;19(Suppl 1):2-5.

25. Dawes P, Emsley R, Cruickshanks KJ, et al. Hearing loss and cognition: the role of hearing AIDS, social isolation and depression. PLoS One 2015;10.

26. Ghiringhelli R, lorio MC. Hearing aids and recovery times: a study according to cognitive status. Braz J Otorhinolaryngol 2013;79:177-84.

27. Fritze T, Teipel Óvári A, et al. Hearing Impairment Affects Dementia Incidence. An Analysis Based on Longitudinal Health Claims Data in Germany. PLoS One 2016;11 https://doi.org/

28. Prince M, Wimo A, Guerchet M, et al. World Alzheimer Report. London: Alzheimer's Disase International, 2015.

29. McGilton KS, Höbler F, Campos J, et al. Hearing and vision screening tools for long-term care residents with dementia: protocol for a scoping review. BMJ Open 2016:6:e011945.

30. Bowen M, Edgar DF, Hancock B, et al; The Prevalence of Visual Impairment in People with Dementia (the PrOVIDe study): a cross sectional study of 60-89 year old people with dementia and qualitative exploration of individual, carer and professional perspectives. Health Services and Delivery Research. Southampton: National Institute of Health Research, 2016. No. 4.21

31. Smith SC, Lamping DL, Banerjee S, et al. Measurement of healthrelated quality of life for people with dementia: development of a new instrument (DEMQOL) and an evaluation of current methodology. Health Technol Assess 2005;9:1-93.

32. Ogden J, Hills L. Understanding sustained behavior change: the role of life crises and the process of reinvention. Health 2008;12:419-37.

33. Michie S, van Stralen MM, West R. The behaviour change wheel: a new method for characterising and designing behaviour change interventions. Implement Sci 2011;6:42.

34 Ventry IM, Weinstein BE. The hearing handicap inventory for the elderly: a new tool. Ear Hear 1982;3:128-34.
35 McKhann G, Drachman D, Folstein M, et al. Clinical diagnosis of Alzheimer's disease: report of the NINCDS-ADRDA Work Group under the auspices of Department of Health and Human Services Task Force on Alzheimer's Disease. Neurology 1984;34:939-44.

36 Nasreddine ZS, Phillips NA, Bédirian V, et al. The Montreal Cognitive Assessment, MoCA: a brief screening tool for mild cognitive impairment. J Am Geriatr Soc 2005;53:695-9.

37 Sivantos Hearcheck, Siemens. https://www.bestsound-technology. co.uk/nhs/equipment/hear-check/

38 Peek Vision. http://www.peekvision.org/

39 Broadway DC. Visual field testing for glaucoma - a practical guide. Community Eye Health 2012:25:66-80.

40 Craig P, Dieppe P, Macintyre S, et al. Developing and evaluating complex interventions: the new Medical Research Council guidance. BMJ 2008;337:a1655.

41 Leroi I, Pye A, Armitage CJ, et al. Research protocol for a complex intervention to support hearing and visual function to improve the lives of people with dementia. Pilot and Feasibility Studies 2017.

42 Dawes P, Wolski L, Himmelsbach I, et al. Interventions for hearing and vision impairment to improve outcomes for people with dementia: a scoping review. Int Psychogeriatr 2017.

43 Newell F. Framework for patient and public participation in primary care commissioning. NHS England $2016 \mathrm{https} / / / \mathrm{www}$.england.nhs. uk/commissioning/wp-content/uploads/sites/12/2016/03/framwrkpublic-partcptn-prim-care.pdf

44 Essilor International. https://www.essilor.com/en/

45 Starkey Hearing Technologies. http://www.starkey.co.uk/

46 Lawrence V, Murray J, Banerjee S, et al; The experiences and needs of people with dementia and serious visual impairment: $A$ qualitative study. Thomas Pocklington Trust. London: Thomas Pocklington Trust, 2008. occasional paper 16.

47 Bucks RS, Ashworth DL, Wilcock GK, et al. Assessment of activities of daily living in dementia: development of the Bristol Activities of Daily Living Scale. Age Ageing 1996;25:113-20.

48 Whitson HE, Whitaker D, Potter G, et al. A low-vision rehabilitation program for patients with mild cognitive deficits. JAMA Ophthalmol 2013;131:912-9.

49 Clare L, Bayer A, Burns A, et al. Goal-oriented cognitive rehabilitation in early-stage dementia: study protocol for a multicentre single-blind randomised controlled trial (GREAT). Trials 2013:14:1-15.

50 Watermeyer TJ, Hindle JV, Roberts J, et al. Goal Setting for Cognitive Rehabilitation in Mild to Moderate Parkinson's Disease Dementia and Dementia with Lewy Bodies. Parkinsons Dis 2016;2016.

51 Fritze T, Teipel S, Óvári A, et al. Hearing Impairment Affects Dementia Incidence. An Analysis Based on Longitudinal Health Claims Data in Germany. PLoS One 2016;11:e0156876 https://doi.org/

52 Gannon B. The feasibility study: a health economics perspective. Glob Reg Health Technol Assess 2017;4:e65-8.

53 Mayring P, Qualitative Content Analysis. Forum Qualitative Sozialforschung / Forum: Qualitative Social Research, 1:2 http://nbnresolving.de/urn:nbn:de:0114-fqs0002204

54 MAXQDA. QSR International Pty Ltd, Doncaster, Victoria, Australia). http://www.maxqda.com/

55 EuroQol Group. EuroQol-a new facility for the measurement of health-related quality of life. Health Policy 1990;16:199-208.

56 Brazier JE, Roberts J. The estimation of a preference-based measure of health from the SF-12. Med Care 2004;42:851-9.

57 Wimo A, Gustavsson A, Jonsson L. Application of Resource Utilization in Dementia (RUD) instrument in a global setting. Alzheimers Dement 2013;9:429-435.

58 Tschanz JT, Norton MC, Zandi PP, et al. The Cache County Study on Memory in Aging: factors affecting risk of Alzheimer's disease and its progression after onset. Int Rev Psychiatry 2013;25:673-85.

59 Lyketsos CG, Lopez O, Jones B, et al. Prevalence of neuropsychiatric symptoms in dementia and mild cognitive impairment: results from the cardiovascular health study. JAMA 2002;288:1475-83.

60 Kroenke K, Spitzer RL, Williams JB. The PHQ-15: validity of a new measure for evaluating the severity of somatic symptoms. Psychosom Med 2002;64:258-66.

61 Schofield HL, Murphy B, Herrman HE, et al. Family caregiving: measurement of emotional well-being and various aspects of the caregiving role. Psychol Med 1997;27:647-57.

62 Yesavage JA. Geriatric depression scale. Psychopharmacology Bulletin 1988:24:709-11.

63 Chen G, Gully SM, Eden D. Validation of a New General Self-Efficacy Scale. Organizational Research Methods 2001;4:62-83.

64 Stelmack JA, Massof RW. Using the VA LV VFQ-48 and LV VFQ-20 in low vision rehabilitation. Optom Vis Sci 2007;84:705-9.

65 Cummings JL. The Neuropsychiatric Inventory: assessing psychopathology in dementia patients. Neurology 1997;48:S10-S16. 
66 Burns D, Scale RS. Ten Days to Self-Esteem 1999. New York: Harper Collins Publishers, 1983.
67 Gatehouse S. Glasgow Hearing Aid Benefit Profile: Derivation and Validation of a Client-centered Outcome Measure for Hearing Aid Services. J Am Acad Audiol 1999;10:80-103. 